Y. WATANABE

KODAI MATH. J.

6 (1983), 100-109

\title{
THE SECTIONAL CURVATURE OF A 5-DIMENSIONAL HARMONIC RIEMANNIAN MANIFOLD
}

\author{
By Yoshiyuki Watanabe
}

In 1939, E. T. Copson and H.S. Ruse [4] initiated to study harmonic Riemannian manifolds. And, in 1944, A. Lichnerowicz [13] proved the curvature identities (cf. §2) in a harmonic Riemannian manifold and gave the following

Conjecture. If a Riemannian manifold $M$ with positive definite metric is harmonic, then $M$ is locally symmetric.

For Riemannian manifolds of dimension 2 or 3 the conjecture is trivially affirmative, because a harmonic manifold is Einsteinian and therefore of constant curvature. A.G. Walker [22] showed that the conjecture is affirmative for Riemannian manifolds of dimension 4 (cf. [16], [2]). Later, harmonic Riemannian manifolds were studied by T.J. Willmore [28], [29], A. J. Ledger [11], [12], A. Allamigeon [1], S. Tachibana [19], Y. Watanabe [23], [24], [25], [26], [27], A. Besse [2], L. Vanhecke [20], [21], M. Kôzaki [9], K. Sakamoto [18] and others. But it is an open problem to show that the Lichnerowicz's conjecture is affirmative for Riemannian manifolds of dimension $>4$, and no counterexample is known up to now. The main purpose of this paper is to prove the main Theorem 3.3 giving a sufficient condition, by pinching the sectional curvature at a point, for a harmonic Riemannian manifold of dimension 5 to be locally symmetric, i.e. of constant curvature. In $\S 3$, we prove Lemma 3.2 , which implies immediately the main theorem, because a locally symmetric harmonic manifold is locally flat or locally isometric to a rank one symmetric space (cf. [12], [6], [3]), i. e., because it is of constant curvature in the case where it is odd-dimensional.

In $\S 1$, we give some preliminaries concerning Riemannian manifolds. In $\S 2$, we give definitions and curvature conditions concerning harmonic Riemannian manifolds. The last section $\S 3$ is devoted to the proof of the main theorem and another.

The author wishes to express his sincere thanks to Professor S. Ishihara, who gave him many valuable suggestions and guidances. He also would like to acknowledge Professor S. Tachibana and Professor K. Takamatsu for their continuing guidances and encouragements.

Received April 28, 1982 


\section{$\S 1$. Preliminaries.}

First, we shall recall in Riemannian manifolds some curvature identities which will be useful in the sequel. In the present paper, every Riemannian manifold we consider is assumed to be of class $C^{\omega}$ and connected. Let $M$ be an $n$-dimensional Riemannian manifold with positive definite metric $g$ and $\nabla$ be the Levi-Civita connection. The Riemannian curvature tensor $R$ is defined by

$$
R(X, Y) Z=\nabla_{X} \nabla_{Y} Z-\nabla_{Y} \nabla_{X} Z-\nabla_{[X, Y]} Z
$$

for any vector fields $X, Y$ and $Z$. With local components the curvature tensor can be written as $R=\left(R_{k j i}{ }^{h}\right)$. Let $R_{1}=\left(R_{a j i}{ }^{a}\right)=\left(R_{j i}\right)$ and $S=g^{j i} R_{j i}$ be the Ricci tensor and the scalar curvature, respectively. Denote by $T_{x} M$ the tangent space to $M$ at a point $x$ of $M$. The sectional curvature $\kappa_{x}(X, Y)$ for an orthonormal pair $\{X, Y\}$, where $X$ and $Y$ belonging to $T_{x} M$, is given by

$$
\kappa_{x}(X, Y)=-g(R(X, Y) X, Y) .
$$

We recall the well known Bianchi's identities

$$
\begin{aligned}
& \text { (a) } R_{k j i}{ }^{h}+R_{j i k}{ }^{h}+R_{\imath k \jmath}{ }^{h}=0, \\
& \text { (b) } \nabla_{l} R_{k j i}{ }^{h}+\nabla_{k} R_{j l \imath}{ }^{h}+\nabla_{j} R_{l k \imath}{ }^{h}=0,
\end{aligned}
$$

where $\nabla_{l}$ denotes the covariant differentiation with respect to the Levi-Civita connection. Generally speaking, we put $|T|^{2}=T_{k j i} T^{k j i}$ for any tensor field of any type, say $T=\left(T_{k j i}\right)$. Then from (1.1) we get the following well known formulas (cf. [17])

$$
\begin{aligned}
\nabla^{u} R^{a b c d} \nabla^{u} R_{a d c b} & =\nabla_{u} R^{a b c d} \nabla_{u} R_{c b a d}=\nabla^{u} R^{a b c d} \nabla_{c} R_{a b u d} \\
& =\nabla^{u} R^{a b c d} \nabla_{d} R_{a b c u}=\nabla^{u} R^{a b c d} \nabla_{a} R_{u b c d} \\
& =\nabla^{u} R^{a b c d} \nabla_{c} R_{u b a d}=\frac{1}{2}|\nabla R|^{2} .
\end{aligned}
$$

On putting (cf. [2])

$$
\hat{R}=R^{a b c d} R_{a b}{ }^{u v} R_{c d u v} \text { and } \hat{R}=R^{a b c d} R_{a}{ }^{u}{ }^{v}{ }^{v} R_{b u d v} \text {, }
$$

we have the following formulas (cf. T. Sakai [17]):

$$
\begin{aligned}
& \text { (a) } \quad R^{a b c d} R_{a b}{ }^{u v} R_{c u d v}=R^{a b c d} R_{a}{ }^{u}{ }^{v} R_{c d u v}=R^{a b c d} R_{a c}{ }^{u v} R_{b d u v}=\frac{1}{2} \hat{R} \text {, } \\
& \text { (b) } \quad R^{a b c d} R_{a}{ }^{u}{ }^{v} R_{c u d v}=R^{a b c d} R_{a}{ }^{u}{ }^{v} R_{c v u d}=R^{a b c d} R_{a}{ }^{u}{ }_{c}{ }^{v} R_{b d u v} \\
& =R^{a b c d} R_{a c}{ }^{u v} R_{b u d v}=\frac{1}{4} \hat{R}, \\
& \text { (c) } R^{a b c d} R_{a}{ }^{u}{ }_{c}^{v} R_{b v d u}=R^{a b c d} R_{a}{ }^{v}{ }^{u} R_{b u d v}=\stackrel{R}{R}-\frac{1}{4} \hat{R} \text {. }
\end{aligned}
$$


A. Lichnerowicz [14] gave the following identity

$$
\frac{1}{2} \Delta|R|^{2}=|\nabla R|^{2}-4 R^{j i h k} \nabla_{\jmath} \nabla_{h} R_{\imath k}+2 R_{\imath \jmath} R^{i h k l} R^{\jmath}{ }_{h k l}+\hat{R}+4 \stackrel{R}{R},
$$

where $\Delta$ is the Laplace-Beltrami operator acting on differentiable functions on $M$ (cf. [30]).

If $M$ is Einsteinian and $|R|^{2}=$ constant, then (1.4) reduces to

$$
|\nabla R|^{2}+\frac{2}{n} S|R|^{2}+\hat{R}+4 \stackrel{R}{=}=0 .
$$

\section{§2. Harmonic Riemannian manifolds.}

Let $M$ be a Riemannian manifold of dimension $n$. Take a normal neighborhood $N$ centered at a point $x_{0}$ of $M$. Denoting by $s(x)$ the geodesic distance measured from $x_{0}$ to a point $x$ of $N$, we define in $N$ a function $s$ by $s: x \rightarrow$ $s(x)(x \in N)$. Given a fixed point $x_{0}$ of $M$, the Riemannian manifold $M$ is said to be harmonic at the point $x_{0}$, if there is a normal neighborhood $U$ centered at $x_{0}$ in such a way that there is in $U-\left\{x_{0}\right\}$ a nontrivial solution $u$, analytically depending only on $\Omega=(1 / 2) s^{2}$, of the Laplace equation $\Delta u=0$. When $M$ is harmonic at every point of $M$, it is called a harmonic Rıemannian manifold. It is well known (cf. [13], [16]) that in a harmonic Riemannian manifold the local function $\Delta \Omega$ has the form $f(\Omega)$ in each normal neighborhood $U$, where $f(\Omega)$ is a function analytically depending on $\Omega$, and the function $f(\Omega)$ is independent of the choice of the center $x_{0}$. The function $f(\Omega)$ is called the characteristic function of the harmonic Riemannian manifold.

Let $M$ be a harmonic Riemannian manifold of dimension $n$ and $\left\{y^{i}\right\}$ be a normal coordinate system, covering a sufficient small normal neighborhood $U$ centered at a point $x_{0}$ of $M$. Then $\Delta \Omega=f(\Omega)$ and $f(\Omega)$ thus admits the Maclaurin expansion

$$
\begin{aligned}
f(\Omega) & =f(0)+\dot{f}(0) \Omega+\frac{1}{2 !} \ddot{f}(0) \Omega^{2}+\frac{1}{3 !} \ddot{f}(0) \Omega^{3}+\cdots \\
& =f(0)+\frac{1}{2} \dot{f}(0) s^{2}+\frac{1}{2 ! 2^{2}} \ddot{f}(0) s^{4}+\frac{1}{3 ! 2^{3}}-\ddot{f}(0) s^{6}+\cdots,
\end{aligned}
$$

taking account of $\Omega=(1 / 2) s^{2}$, where $(\cdot)$ means the operator taking the derivative with respect to $\Omega$. On the other hand, in any Riemannian manifold the formula

$$
\Delta \Omega=n+\left\{\begin{array}{c}
\imath \\
\imath k
\end{array}\right\} y^{k}
$$

holds with respect to normal coordinates $\left\{y^{k}\right\}$, where $\left\{\begin{array}{c}i \\ j k\end{array}\right\}$ denotes the Christoffel symbols. If $\left\{\begin{array}{c}\imath \\ l k\end{array}\right\}$ are expanded in Taylor expansion with respect to $y^{h}$, then 
using (2.1) and (2.2), the following curvature conditions are obtained (cf. [13], [10], [16]):

$$
\begin{gathered}
R_{j i}=-\frac{3}{2} \dot{f}(0) g_{\jmath i}, \quad S=-\frac{3 n}{2} \dot{f}(0), \\
\Im\left(R_{p \imath j}{ }^{q} R_{q k l}{ }^{p}+\frac{45}{8} \ddot{f}(0) g_{\imath \jmath} g_{k l}\right)=0, \\
\Im\left(9 \nabla_{k} R_{p \imath \jmath}{ }^{q} \nabla_{l} R_{q m n}{ }^{p}-32 R_{p \imath j}^{q} R_{q k l}{ }^{r} R_{r m n}{ }^{p}-315 \ddot{f}(0) g_{\imath \jmath} g_{k l} g_{m n}\right)=0,
\end{gathered}
$$

where $\subseteq$ means the summation taken over all permutation of the free indices appearing incide the parenthesis ( ). By the definition of harmonicity we see that $\dot{f}(0), \ddot{f}(0)$ and $\ddot{f}(0)$ are absolute constants, i. e. that they are independent of choice of the center $x_{0}$. Then transvecting $g^{2 j} g^{k l}$ with (2.4) and using (2.3), we obtain

$$
|R|^{2}=-\frac{3 n}{2}\left\{\dot{f}(0)^{2}+\frac{5(n+2)}{2} \ddot{f}(0)\right\}
$$

and see that $|R|^{2}$ is constant.

We now need the following two lemmas for computing $\dddot{f}(0)$ in terms of the scalar functions constructed by the curvature tensors.

Lemma 2.1. For a tensor ( field) $T=\left(T_{\imath j k l m n}\right)$ of type $(0,6)$, we have

$$
\begin{aligned}
& g^{\imath \jmath} g^{k l} g^{m n} \subseteq\left(T_{\imath j k l m n}\right)=48\left(T^{\imath}{ }_{i{ }^{j}{ }^{k}{ }_{k}}+T^{\jmath}{ }_{i}{ }^{j k}{ }_{j k}+T^{\imath{ }^{j}{ }_{k j}}+T^{\imath \jmath}{ }_{\imath \jmath}{ }_{k}{ }_{k}+T^{\imath \jmath}{ }_{\imath{ }_{j k}}\right. \\
& +T^{\imath \jmath}{ }_{\imath k}^{k}{ }_{k j}+T^{\imath \jmath}{ }_{j i}{ }_{k}+T^{\imath \jmath}{ }_{j}{ }_{\imath k}+T^{\imath \jmath}{ }_{j k}{ }_{2}+T^{\imath j k}{ }_{\imath \jmath k} \\
& \left.+T^{\imath j k}{ }_{\imath k \jmath}+T^{\imath j k}{ }_{j i k}+T^{\imath j k}{ }_{j k i}+T_{k \imath j}^{\imath j k}+T_{k j \imath}^{\imath j k}\right) \text {. }
\end{aligned}
$$

Proof. See [23].

LEMMA 2.2. In a harmonic Riemannian manfold, we have

where
(a) $g^{\imath \jmath} g^{k l} g^{m n} \subseteq\left(A_{\imath j k l m n}\right)=144|\nabla R|^{2}$,
(b) $g^{\imath \jmath} g^{k l} g^{m n} \widetilde{S}\left(B_{\imath j k l m n}\right)=48\left(\frac{S^{3}}{n^{2}}+\frac{9}{2 n} S|R|^{2}-\frac{7}{2} \hat{R}+\stackrel{R}{R}\right)$,
(c) $g^{\imath \jmath} g^{k l} g^{m n} \mathbb{S}\left(g_{\imath j} g_{k l} g_{m n}\right)=48 n(n+2)(n+4)$,

$$
A_{\imath j k l m n}=\nabla_{k} R_{p \imath j}{ }^{q} \nabla_{l} R_{q m n}^{p} \quad \text { and } \quad B_{\imath j k l m n}=R_{p l j}^{q} R_{q k l}{ }^{r} R_{r m n}^{p} .
$$

Proof. Putting $T_{\imath j k l m n}=g_{\imath} g_{k \imath} g_{m n}$ in Lemma 2.1, the formula (c) follows immediately. Next putting $T_{\imath \jmath k l m n}=A_{\imath \jmath k l m n}$ in Lemma 2.1, (1.1), (1.2) and (2.3) imply that

$$
A^{\imath j k}{ }_{\imath j k}=\frac{1}{2}|\nabla R|^{2}, \quad A^{\imath j k}{ }_{\imath k \jmath}=\frac{1}{4}|\nabla R|^{2}, \quad A^{\imath j k}{ }_{j i k}=|\nabla R|^{2},
$$




$$
A_{\jmath k \imath}^{\imath j k}=\frac{1}{2}|\nabla R|^{2}, \quad A_{k \imath \jmath}^{\imath j k}=\frac{1}{2}|\nabla R|^{2}, \quad A_{k \jmath \imath}^{\imath j k}=\frac{1}{2}|\nabla R|^{2},
$$

and all the others corresponding to the terms appearing in the right hand side of (2.7) vanish. Thus we obtain the formula (a). Lastly putting $T_{\imath j k l m n}=B_{\imath j k l m n}$ in Lemma 2.1, (1.1), (1.3) and (2.3) imply

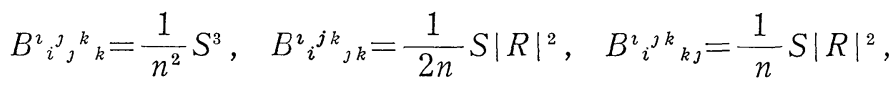

$$
\begin{aligned}
& B^{\imath \jmath}{ }_{\imath \jmath}{ }_{k}=\frac{1}{2 n} S|R|^{2}, \quad B^{\imath \jmath_{\imath}{ }_{j k}}=-\frac{1}{4} \hat{R}, \quad B^{\imath \jmath}{ }^{k}{ }_{k \jmath}=-\frac{1}{2} \hat{R}, \\
& B^{\imath \jmath}{ }_{\jmath i}{ }_{k}=\frac{1}{n} S|R|^{2}, \quad B^{\imath \jmath}{ }_{\jmath}{ }_{\imath k}=-\frac{1}{2} \hat{R}, \quad B^{\imath \jmath}{ }_{j}{ }_{k \imath}=-\hat{R}, \\
& B_{\imath \jmath k}^{\imath j k}=\stackrel{R}{R}-\frac{1}{4} \hat{R}, \quad B_{\imath \imath_{\imath \jmath}}^{\imath j k}=-\frac{1}{4} \hat{R}, \quad B^{\imath j k}{ }_{j \imath k}=-\frac{1}{4} \hat{R}, \\
& B_{j k \imath}^{\imath j k}=-\frac{1}{2} \hat{R}, \quad B_{k \imath \jmath}^{\imath j k}=-\frac{1}{2 n} S|R|^{2}, \quad B_{k \jmath i}^{\imath \jmath k}=\frac{1}{n} S|R|^{2} .
\end{aligned}
$$

Thus we obtain the formula (b).

Remark. Recently A. Gray and L. Vanhecke [7] has given in a Riemannian manifold many formulas which are useful in obtaining systematically scalar functions such as given in Lemma 2.2.

If we transvect $g^{\imath \jmath} g^{k l} g^{m n}$ with (2.5), then taking account of the formulas (2.8), we have the following lemma (cf. Y. Watanabe [23]).

LEMma 2.3. In a harmonic Riemannian manifold of dimension $n$, we have

$$
27|\nabla R|^{2}-32\left(\frac{S^{3}}{n^{2}}+\frac{9}{2 n} S|R|^{2}-\frac{7}{2} \hat{R}+\stackrel{R}{R}\right)=315 n(n+2)(n+4) \ddot{f}(0) .
$$

\section{$\S 3$. 5-dimensional harmonic Riemannian manifolds.}

We shall now prove a curvature identity (3.4) in a harmonic Riemannian manifold $M$ of dimension 5 . To do so, we introduce in a Riemannian manifold of dimension $n$ a function $G_{(m)}$ by

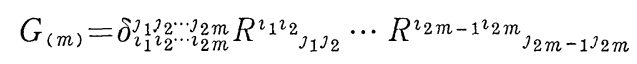

for any natural number $m \geqq 1$, where

$$
\delta_{\imath_{1} i_{2} \cdots i_{2 m}}^{\jmath_{1} \gamma_{2} \cdots}=\left|\delta_{\imath_{b}}^{\jmath} a\right| \quad(a, b=1,2, \cdots, 2 m)
$$

is the so-called generalized Kronecker delta (cf. [15]). When $M$ is compact and $n=2 m$, the $G_{(m)}$ is, as is well known, the integrand of the Gauss-Bonnet 
theorem (see, for example, S. Kobayashi and K. Nomizu [8]). However, the function $G_{(m)}$ vanishes identically for a Riemannian manifold $M$ of dimension $n<2 m$, because in such a case $\delta_{i_{1} i_{2} \cdots i_{2} m}^{\gamma_{1} \lambda_{2} \cdots j_{2} m}$ is equal to zero. Therefore if the dimension of $M$ is 5 , then

$$
G_{(3)}=0 \text {, }
$$

which will be used in the sequel.

On the other hand, the function $G_{(3)}$ has in a Riemannian manifold of dimension $n$ the following form (cf. [17], [5]):

$$
\begin{aligned}
G_{(3)}= & 8 \stackrel{R}{R}-4 \hat{R}-24 R^{a b} R^{c d} R_{a b c d}-24 R^{u v} R_{u}^{a b c} R_{v a b c} \\
& +16 R^{a b} R_{a}^{c} R_{b c}+S^{3}-12 S\left|R_{1}\right|^{2}+3 S|R|^{2},
\end{aligned}
$$

as a consequence of (1.3). Thus using (3.3), we see that in an Einsteinian manifold of dimension 5 the formula (3.2) reduces to

$$
4 \stackrel{R}{R}-2 \hat{R}=\frac{S}{10}\left(9|R|^{2}-S^{2}\right) .
$$

The identity (3.4) is also obtained by transvecting $R^{h \imath j k}$ with the identity

$$
\begin{gathered}
R_{h \imath p q} R_{j k}^{p q_{j}}+2 R_{h p q k} R_{j}{ }^{p q_{i}}-2 R_{h p q j} R_{k}{ }_{i}+\frac{3}{5} S R_{h \imath j k} \\
=\left(\frac{3}{20}|R|^{2}-\frac{1}{20} S^{3}\right)\left(g_{h j} g_{\imath k}-g_{h k} g_{\imath j}\right),
\end{gathered}
$$

proved by E.M. Patterson [15] in a harmonic Riemannian manifold of dimension 5 .

From now on, let $M$ be a 5-dimensional harmonic Riemannian manifold. We note here that any harmonic Riemannian manifold is necessarily Einsteinian (see $\S 2$ ). Eliminating $\stackrel{R}{R}$ from (1.5) and (3.4), we have

$$
|\nabla R|^{2}+\frac{13}{10} S|R|^{2}+3 \hat{R}-\frac{S^{3}}{10}=0 .
$$

Eliminating $\stackrel{R}{R}$ from $(2.9)$ and (3.4), we have

$$
27|\nabla R|^{2}+96 \hat{R}-36 S|R|^{2}-\frac{12}{25} S^{3}=315^{2} \ddot{f}(0) .
$$

Next eliminating $\hat{R}$ from (3.5) and (3.6), we have

$$
5|\nabla R|^{2}+\frac{388}{5} S|R|^{2}-\frac{68}{25} S^{3}=-315^{2} \ddot{f}(0) .
$$

Since $|R|^{2}$ and $S$ are constant in a harmonic Riemannian manifold because of (2.3) and (2.6), we have from (3.7), (3.5) and (3.4)

Proposition 3.1. In a 5-dimensional harmonic Riemannian manifold, the 
scalar functions $|\nabla R|^{2}, \hat{R}$ and $\stackrel{R}{R}$ are all constant.

In a harmonic Riemannian manifold $M$ of dimension 5 , we take a fixed point $x_{0}$ and a fixed unit vector $X$ belonging to the tangent space $T_{0} M$ to $M$ at $x_{0}$. Transvecting $X^{k} X^{i} X^{j} X^{l} X^{m} X^{n}$ with (2.5), we have at the point $x_{0}$

$$
9 \nabla_{k} R_{p \imath j}{ }^{q} \nabla_{l} R_{q m n}{ }^{p} X^{k} X^{i} X^{j} X^{l} X^{m} X^{n}=32 R_{p \imath \jmath}^{q} R_{q k l}^{r} R_{r m n}{ }^{p} X^{k} X^{i} X^{j} X^{l} X^{m} X^{n}+315 \ddot{f}(0) .
$$

Substituting $\ddot{f}(0)$ given by (3.7) into the equation above, we have at the point $x_{0}$ the following key equation

$$
\begin{aligned}
& 9 \nabla_{k} R_{p \imath \jmath}{ }^{q} \nabla_{l} R_{q m n}{ }^{p} X^{k} X^{i} X^{j} X^{l} X^{m} X^{n}+\frac{1}{63}|\nabla R|^{2} \\
& \quad=32 R_{p \imath j}{ }^{q} R_{q k l}{ }^{r} R_{r m i}{ }^{p} X^{k} X^{i} X^{j} X^{l} X^{m} X^{n}+\frac{1}{315}\left(-\frac{388}{5} S|R|^{2}+\frac{68}{25} S^{3}\right) .
\end{aligned}
$$

We now define a linear transformation $\Pi_{X}$ in the tangent space $T_{0} M$ by

$$
\Pi_{X}(Y)=-R(X, Y) X
$$

for $Y \in T_{0} M$, which implies immediately

$$
\Pi_{X}(X)=0 .
$$

For simplicity, we put $\Pi_{X}=\Pi$. Then $X$ is obviously an eigen vector of $\Pi$ with eigen value 0 , because of (3.10). Since the linear transformation $\Pi$ is symmetric because of (3.9), there is an orthonormal basis $\left\{X, e_{1}, e_{2}, e_{3}, e_{4}\right\}$ such that

$$
\Pi\left(e_{\alpha}\right)=\lambda_{\alpha} e_{\alpha} \quad(\alpha=1,2,3,4) .
$$

Then by (2.3) and (2.4) we get

$$
\begin{aligned}
& \operatorname{Tr}(\Pi)=R_{j i} X^{j} X^{i}=\frac{S}{5}=-\frac{3}{2} \dot{f}(0), \\
& \operatorname{Tr}\left(\Pi^{2}\right)=R_{p \imath \jmath}{ }^{q} R_{q k l}{ }^{p} X^{i} X^{\jmath} X^{k} X^{l}=-\frac{45}{8} \ddot{f}(0), \\
& \operatorname{Tr}\left(\Pi^{3}\right)=R_{p \imath \jmath}^{q} R_{q k l}{ }^{r} R_{r m n}{ }^{p} X^{i} X^{j} X^{k} X^{l} X^{m} X^{n},
\end{aligned}
$$

where $\operatorname{Tr}$ means the trace of each of linear transformations $\Pi, \Pi^{2}=\Pi \circ \Pi$ and $\Pi^{3}=\Pi \circ \Pi \circ \Pi$. Thus (3.8) implies the following equation (3.13) at the point $x_{0}$ because of (2.6) and (3.11).

$$
\begin{aligned}
& 9 \nabla_{k} R_{p \imath \jmath}{ }^{q} \nabla_{l} R_{q m n}{ }^{p} X^{k} X^{i} X^{j} X^{l} X^{m} X^{n}+\frac{1}{63}|\nabla R|^{2} \\
& \quad=32 \operatorname{Tr}\left(\Pi^{3}\right)+\frac{1}{315}\left\{-388 \operatorname{Tr}(\Pi)\left(-\frac{15}{2} \dot{f}(0)^{2}-\frac{525}{4} \ddot{f}(0)\right)+340(\operatorname{Tr}(\Pi))^{3}\right\} .
\end{aligned}
$$

Then arranging (3.13) and using (3.12), we get at the point $x_{0}$ 


$$
9 \nabla_{k} R_{p \imath}{ }^{q} \nabla_{l} R_{q m n}{ }^{p} X^{k} X^{i} X^{j} X^{l} X^{m} X^{n}+\frac{1}{63}|\nabla R|^{2}={ }_{27}^{4} F(\lambda),
$$

where

$$
F(\lambda)=216 \operatorname{Tr}\left(\Pi^{3}\right)+35(\operatorname{Tr}(\Pi))^{3}-194 \operatorname{Tr}(\Pi) \operatorname{Tr}\left(\Pi^{2}\right) .
$$

As a consequence of (3.11), we have

$$
\begin{aligned}
F(\lambda) & =216 \sum_{\alpha} \lambda_{\alpha}^{3}+35\left(\sum_{\alpha} \lambda_{\alpha}\right)^{3}-194\left(\sum_{\alpha} \lambda_{\alpha}\right) \sum_{\beta} \lambda_{\beta}^{2} \\
& =54\left\{4 \sum_{\alpha} \lambda_{\alpha}^{3}-\left(\sum_{\alpha} \lambda_{\alpha}\right) \sum_{\beta} \lambda_{\beta}^{2}\right\}+35 \sum_{\alpha} \lambda_{a}\left\{\left(\sum_{\beta} \lambda_{\beta}\right)^{2}-4 \sum_{\beta} \lambda_{\beta}^{9}\right\} \\
& =54 \sum_{\beta<r}\left(\lambda_{\beta}-\lambda_{\gamma}\right)^{2}\left(\lambda_{\beta}+\lambda_{\gamma}\right)+35\left(\sum_{\alpha} \lambda_{\alpha}\right) \sum_{\beta<\gamma}\left(\lambda_{\beta}-\lambda_{i}\right)^{2} \\
& =\sum_{\beta<\gamma}\left(\lambda_{\beta}-\lambda_{\gamma}\right)^{2}\left\{54\left(\lambda_{\beta}+\lambda_{i}\right)-35 \sum_{\alpha} \lambda_{\alpha}\right\}
\end{aligned}
$$

which implies the following formulas

$$
\begin{aligned}
F(\lambda)= & \left(\lambda_{1}-\lambda_{2}\right)^{2}\left\{19\left(\lambda_{1}+\lambda_{2}\right)-35\left(\lambda_{3}+\lambda_{4}\right)\right\}+\left(\lambda_{1}-\lambda_{3}\right)^{2}\left\{19\left(\lambda_{1}+\lambda_{3}\right)-35\left(\lambda_{2}+\lambda_{4}\right)\right\} \\
& +\left(\lambda_{1}-\lambda_{4}\right)^{2}\left\{19\left(\lambda_{1}+\lambda_{4}\right)-35\left(\lambda_{2}+\lambda_{3}\right)\right\}+\left(\lambda_{2}-\lambda_{3}\right)^{2}\left\{19\left(\lambda_{2}+\lambda_{3}\right)-35\left(\lambda_{1}+\lambda_{1}\right)\right\} \\
& +\left(\lambda_{2}-\lambda_{4}\right)^{2}\left\{19\left(\lambda_{2}+\lambda_{4}\right)-35\left(\lambda_{1}+\lambda_{3}\right)\right\}+\left(\lambda_{3}-\lambda_{4}\right)^{2}\left\{19\left(\lambda_{3}+\lambda_{4}\right)-35\left(\lambda_{1}+\lambda_{2}\right)\right\}
\end{aligned}
$$

and

$$
\begin{aligned}
F(\lambda)= & \left(\lambda_{1}-\lambda_{2}\right)^{2}\left\{19 \sum_{\alpha} \lambda_{\alpha}-54\left(\lambda_{3}+\lambda_{4}\right)\right\}+\left(\lambda_{1}-\lambda_{3}\right)^{2}\left\{19 \sum_{\alpha} \lambda_{\alpha}-54\left(\lambda_{2}+\lambda_{4}\right)\right\} \\
& +\left(\lambda_{1}-\lambda_{4}\right)^{2}\left\{19 \sum_{\alpha} \lambda_{\alpha}-54\left(\lambda_{2}+\lambda_{3}\right)\right\}+\left(\lambda_{2}-\lambda_{3}\right)^{2}\left\{19 \sum_{\alpha} \lambda_{\alpha}-54\left(\lambda_{1}+\lambda_{4}\right)\right\} \\
& +\left(\lambda_{2}-\lambda_{4}\right)^{2}\left\{19 \sum_{\alpha} \lambda_{\alpha}-54\left(\lambda_{1}+\lambda_{3}\right)\right\}+\left(\lambda_{3}-\lambda_{4}\right)^{2}\left\{19 \sum_{\alpha} \lambda_{\alpha}-54\left(\lambda_{1}+\lambda_{2}\right)\right\} .
\end{aligned}
$$

As a consequence of (3.14), we have the following inequality

$$
F(\lambda) \geqq 0 \text {. }
$$

We first note that $\lambda_{\alpha}$ is the sectional curvature for the orthonormal pair $\left\{X, e_{\alpha}\right\} \quad(\alpha=1,2,3,4)$, because of the definition (3.9) of $\Pi=\Pi_{X}$. Suppose that all $\lambda_{\alpha}$ satisfy $\delta \geqq \lambda_{\alpha} \geqq(19 / 35) \delta$ for some $\delta \geqq 0$. Then we see from (3.15) that the right hand side of (3.14) is non-positive. Consequently it follows from (3.14) and (3.17) that $|\nabla R|^{2}=0$ at the point $x_{0}$ of $M$. Since $M$ is connected, Proposition 3.1 implies that $\nabla R=0$, i.e. that $M$ is locally symmetric. Thus, we have the following

LeMma 3.2. Let $M$ be a 5-dimensional harmonc Riemanman manfold all of whose sectional curvatures $\kappa_{x}(X, Y)$ at a pornt $x$ satısfy $\delta \geqq \kappa_{x}(X, Y) \geqq(19 / 35) \delta$ for some $\delta \geqq 0$. Then $M$ is locally symmetric. 
Since a locally symmetric harmonic manifold is locally flat or locally isometric to a rank one symmetric space (cf. [12], [6]), it is of constant curvature if it is odd-dimensional. Thus, Lemma 3.2 implies

THEOREM 3.3. Let $M$ be a 5-dimensional harmonic Riemannian manifold all of whose sectional curvatures $\kappa_{x}(X, Y)$ at a point $x$ satısfy $\delta \geqq \kappa_{x}(X, Y) \geqq(19 / 35) \delta$ for some $\delta \geqq 0$. Then $M$ is of constant curvature.

Similarly using (3.16) and noting $S=5 \sum_{\alpha} \lambda_{\alpha}$, we obtain

THEOREM 3.4. Let $M$ be a 5-dimensional harmonic Riemannan manfold. If all sectional curvatures $\kappa_{x}(X, Y)$ at a point $x$ satisfy $\kappa_{x}(X, Y) \geqq(19 / 540) S$, where $S$ is the scalar curvature, then $M$ is of constant curvature.

\section{BIBLIOGRAPHY}

[1] A. Allamigeon, Propriétés globales des espaces de Riemann harmoniques, Ann. Inst. Fourier 15 (1965), 91-132.

[2] A. L. Besse, Manifolds all of whose geodesics are closed, Ergebnisse der Mathematik, vol. 93, Springer-Verlag, Berlin and New York, 1978.

[3] P. Carpenter, A. Gray and T.J. Willmore, The curvature of Einstein symmetric spaces, to appear in Quart. J. Math. Oxford ser..

[4] E. T. Copson and H.S. Ruse, Harmonic Riemannian space, Proc. Roy. Soc. Edinburgh, 60 (1939-40), 117-133.

[5] J.C. Dessertine, Expressions nouvelles de la formula de Gauss-Bonnet en dimension 4 et 6, CR. Acad. Sc. Paris serie A 273 (1971), 164-167.

[6] J.H. Eshenburg, A note on symmetric and harmonic spaces, J. London Math. Soc., 21 (1980), 541-543.

[7] A. GRay And L. Vanhecke, Riemannian geometry as determined by the volumes of small geodesic balls, Acta Math., 142 (1979), 157-198.

[8] S. Kobayashi and K. Nomizu, Foundations of Differential Geometry, Interscience trac. vol. II, 1969.

[9] M. KôzAKI, On the Euler-Poincare characterıstic of 6-dimensıonal harmonic manifold, Math. J. Okayama Unıv., 22 (1981), 85-90.

[10] A. J. LEDGER, Harmonic Riemannian Spaces, Thesis submitted for the degree of Ph. D. University of Durham (1954).

[11] A. J. Ledger, Harmonic homogeneous spaces of Lie groups, J. London Math. Soc., 29 (1954), 345-347.

[12] A. J. Ledger, Symmetric harmonic spaces, J. London Math. Soc. 32 (1957), 53-56.

[13] A. Lichnerowicz, Sur les espaces Riemanniens complètement harmonıques, Bull. Soc. Math. France, 72 (1944), 146-168.

[14] A. Lichnerowicz, Géométrie des groupes de Transformations, Dunod, Paris, 1958.

[15] E. M. Patterson, A class of critical Riemannian metrics, J. London Math. Soc. (2), 23 (1981), 349-358.

[16] H.S. Ruse, A.G. Walker And T.J. Willmore, Harmonic Spaces, Edizionı Cremonese, Roma, 1961. 
[17] T. SAKAI, On eigen-values of Laplacian and curvatures of Riemannian manıfolds, Tôhoku Math. J., 23 (1971), 589-603.

[18] K. Sakamoto, Herical immersions in a unit sphere, to appear.

[19] S. Tachibana, On the characteristic function of spaces of constant holomorphic curvature, Colloq. Math., 26 (1972), 145-155.

[20] L. Vanhecke, A note on harmonic spaces, Bull. Lond. Math. Soc., 13 (1981), 545-546.

[21] L. VAnhecke, A conjecture of Besse on harmonic manifolds, Math. Zeitshurift, 176 (1981), 555-558.

[22] A. G. WALKER, On Lichnerowicz's conjecture for harmonic 4-spaces, J. London Math., 24 (1948-1949), 21-28.

[23] Y. Watanabe, On the characteristic functions of harmonic Kählerian spaces, Tôhoku Math. J., 27 (1975), 13-24.

[24] Y. WATANABE, On the characteristic functions of harmonıc quaternıon Kählerıan spaces, Kōdai Math. Sem. Rep, 27 (1976), 410-420.

[25] Y. WatanABe, On the characteristic functions of quaternion Kählerian spaces of constant Q-sectional curvature, Kōdai Math. Sem. Rep., 28 (1977), 284-299.

[26] Y. Watanabe, The curvature tensors of $S p(2) / S U(2)$ and $S U(5) / S p(2) \times S^{1}$, Kodai Math. J., 5 (1982), 100-110.

[27] Y. Watanabe, Kählerian metrics given by certain potentıal functions, Kodaı Math. J., 5 (1982), 329-338.

[28] T. J. Willmore, Mean-value theorems in harmonic Riemannian spaces, J. London Math. Soc., 25 (1950), 54-57.

[29] T. J. WillmoRE, Some properties of harmonic riemannian manifolds, Convegno di geometia differenziale, Venice 1953 (perrella, Roma 1954), 141-147.

[30] K. YANo, Differential Geometry on complex and Almost complex Spaces, Pergamon, Press, New York, 1965.

\author{
Department of Mathematics \\ FACULTY OF SCIENCE \\ TOYAMa University \\ Gofuku, Toyama, 930, \\ JAPAN
}

